\title{
Reproducible and Opposing Microbiome Signatures Distinguish Autoimmune Diseases and Cancers: A Systematic Review and Meta-Analysis
}

Md Zohorul Islam

Joslin Diabetes Center

Melissa Tran

Joslin Diabetes Center

Tao Xu

Joslin Diabetes Center

Braden T. Tierney

Joslin Diabetes Center

Chirag Patel

Harvard Medical School

Aleksandar David Kostic ( $\square$ Aleksandar.Kostic@joslin.harvard.edu )

Joslin Diabetes Center

Research Article

Keywords: Cancer, autoimmunity, microbiome, metagenome, health.

Posted Date: December 29th, 2021

DOI: https://doi.org/10.21203/rs.3.rs-1195626/v1

License: (c) (i) This work is licensed under a Creative Commons Attribution 4.0 International License.

Read Full License 


\section{Abstract}

Background: The gut microbiome promotes specific immune responses, and in turn the immune system has a hand in shaping the microbiome. Cancer and autoimmune diseases are two major disease families that result from the contrasting manifestations of immune dysfunction. We hypothesized that the opposing immunological profiles between cancer and autoimmunity yield analogously inverted gut microbiome signatures. To test this, we conducted a systematic review and meta-analysis on gut microbiome signatures and their directionality in cancers and autoimmune conditions

Methodology: We searched PubMed, Web of Science, and EMBAS to identify relevant articles to be included in this study. The study was conducted in accordance with Preferred Reporting Items for Systematic Reviews and Meta-Analyses (PRISMA) statements and PRISMA 2009 checklist. Study estimates were pooled by a generic inverse variance random-effects meta-analysis model. The relative abundance of microbiome features was converted to log fold-change and the standard error was calculated from the $p$-values, sample size and fold-change.

Results: We screened 3,874 potentially relevant publications. A total of 82 eligible studies comprising 37 autoimmune and 45 cancer studies with 4,208 healthy human controls and 5,957 disease cases from 27 countries were included in this study. We identified a set of microbiome features that show consistent, opposite directionality between cancers and autoimmune diseases in multiple studies. Fusobacterium and Peptostreptococcus were the most consistently increased genera among the cancer cases which were found to be associated in a remarkable $13(+0.54 \mathrm{log}$ fold-change in 5 studies) and 11 studies (+3.75 log fold-change in 5 studies), respectively. Conversely, Bacteroides was the most prominent genus, which was found to be increased in 12 autoimmune studies ( +0.24 log fold-change in 6 studies) and decreased in six cancer studies (-0.32 log fold-change in 4 studies). Sulfur-metabolism pathways were found to be the most frequent pathways among the member of cancer-increased genus and species.

Conclusions: The surprising reproducibility of these associations across studies and geographies suggests a shared underlying mechanism shaping the microbiome across cancers and autoimmune diseases.

\section{Introduction}

Development of cancer and autoimmune diseases is influenced by contrasting manifestations of immune dysfunction. Immunosubversion is the prime immune functional defect in cancer pathogenesis [1], while hyperactivation of the immune system against self-antigens is the main pathogenic alteration in autoimmune pathogenesis [2]. This contrasting immune landscape between autoimmunity and cancer is also visible at the cellular level. Immune cells modulate the development and progression of cancer and autoimmune conditions. T regulatory cells (Tregs) are the major immune cells found with increased frequency in the tumor-microenvironment and are believed to be negatively associated with antitumor immune responses, promotion of malignancy, and a worsening prognosis in many types of cancers [3- 
5]. Conversely, Treg frequency has been found to be decreased in diverse types of autoimmune diseases such as type 1 diabetes (T1D) [6], arthritis [7], and systemic lupus erythematosus [8-10], which explains the loss of peripheral tolerance in autoimmune pathogenesis [11]. Although genetic background is one of the major predisposing factors both in cancer and autoimmunity, other environmental factors, such as the gut microbiome, have recently been found to be strongly associated with both cancers and autoimmune diseases $[12,13]$. The gut microbiome contributes to the host's "immune education" and lymphoid tissue development $[14,15]$, and is hypothesized to be capable of promoting the immune system, as seen in autoimmune diseases or cancers.

We hypothesized that the gut microbiome would mirror the inverse immunologic relationship between autoimmune disease and cancer. In other words, certain gut microbiome features would be shared across autoimmune diseases and others shared across cancers, with those shared by both conditions demonstrating opposite-sign associations (e.g. depleted in cancer and enriched in autoimmune disease). Although many independent studies have surveyed microbiome associations with autoimmune diseases and cancer, there is a lack of large-scale, evidence-based, systematic analysis of research findings on this topic. The observation of consistent microbiome associations across multiple studies and in diverse geographical locations is paramount to painting the landscape of robust and reproducible microbiome associations in human disease, and comparing associations across diseases is likely to yield novel insights into the underlying and differentiating biology of the phenotypes in question.

We identified 82 published microbiome studies and synthesized the results of the microbiome features that are associated with multiple cancer and autoimmune diseases. Further, we explored the functional potential of the identified gut microbiome features in association with cancer and autoimmunity. These findings revealed a novel set of gut microbiome signatures showing a robust association with multiple cancer or autoimmune disease conditions across multiple studies, which may serve as hypotheses for future studies investigating the relationship between the microbiome and immunological state.

\section{Methods}

\section{Literature source and search strategy}

A systematic database search strategy was employed to identify microbiome studies in cancer and autoimmune diseases. On March 26, 2020, three electronic databases (Pubmed, Web of Science and EMBAS) were searched for relevant publications until the date of the search. The first author (MZI) searched the database. The database search was conducted separately for autoimmune diseases and cancers. The microbiome and disease-related search terms were joined together using the boolean operator "AND" and "OR". Medical Subject Headings (MeSH) terms were used for Pubmed and a combination of general search terms was used for the Web of Science and EMBAS databases. A detailed description of the database search terms is listed in Supplementary Table 1.

\section{Study eligibility criteria}


Studies published in English from 1 January 2008 to 26 March 2020 were considered. The major inclusion criteria included 1) the study was on the association between the gut microbiome with either cancer or autoimmune diseases [i.e, colorectal cancer and adenoma, gastric cancer and adenocarcinoma, pancreatic cancer and ductal adenocarcinoma, prostate cancer, cervical cancer, lung cancer, thyroid papillary cancer, breast cancer, hepatocellular carcinoma, gastrointestinal tract (GIT) neoplasia, Type 1 diabetes, autoimmune arthritis, multiple sclerosis, systemic lupus erythematosus, Graves' disease, primary Sjögren's syndrome, pemphigus vulgaris, anti-N-methyl-D-aspartate receptor (NMDAR) encephalitis, and autoimmune hepatitis], 2) the study subjects were human, 3) the study had clear demarcation of healthy control and disease cases, and 4) the DNA sequencing method was 16s rRNA or shotgun. Cross-sectional, case-control or longitudinal cohort studies were considered to be eligible if they had samples from healthy control and disease cases. Studies were excluded if they had insufficient taxonomic classification below genus, lack statistical comparison of microbiome taxonomic abundance between cases and controls, contained cases that underwent treatment with antibiotics or failed to meet the inclusion criteria mentioned above. We did not include conference abstracts, reports, and experimental and intervention studies.

\section{Study screening and selection}

All publications identified by the systematic database search were first screened for duplicates. After duplicate removal, the titles and abstracts of all publications were independently screened by two authors (MZI and MT) to identify potential studies for full-text screening.

\section{Data extraction}

We developed and evaluated a pilot data extraction spreadsheet before the final data extraction. Full-text articles were screened for data extraction by one author (either MZI and MT). Independent overlapping data extraction was performed for $5 \%$ of the studies. Any disagreements between the data extractors were resolved by consensus. Data were extracted on the first author, year of publication, country, type of disease, method of disease diagnosis, specimen types, number of cases and controls, age of the subject, characteristics of control, DNA sequencing type and platform, availability and source of sequencing data, alpha diversity, statistically significant genus and species and their corresponding $p$-values, the relative abundance of genus and species, and statistically significant metabolic pathways and their corresponding $p$-values.

\section{Maintenance of study standard and quality assessment of individual study}

We conducted this systematic review in accordance with Preferred Reporting Items for Systematic Reviews and Meta-Analyses (PRISMA) statements [16] and PRISMA 2009 checklist (Supplementary Table 2) We did not publish a protocol for this systematic review and meta-analysis. All included studies were independently assessed for quality and bias using Newcastle-Ottawa Scale (NOS)[17]. The risk of bias in each study was assessed in three major domains: study selections, compatibility and ascertainment of exposure. 


\section{Data analysis}

To identify shared and unique microbiome features (genus, species, and metabolic pathways) between autoimmune and cancer studies, we extracted genus, species, and predicted metabolic pathway data that were already analyzed in published studies. We considered all the individual studies as an independent observation and extracted the significant (False discovery rate, FDR adjusted $p$-value $<0.05$ or nominal $p$ value $<0.05$ where FDR adjusted $p$-value was absent) associated microbiome features found between cases and controls. This approach enabled us to exclude spurious microbiome features and to aggregate the most meaningful features from multiple studies. We first removed microbiome features that were found only once in the 82 included studies. We then defined whether a feature is 'increased' or 'decreased' among the cases of cancer and autoimmune studies. We defined a microbiome feature as 'increased in cancer' if it was found to be significantly increased among the cancer cases in more than one cancer study and in at least $70 \%$ of the cancer studies that identify the feature as significant. On the contrary, we considered a microbiome feature as 'decreased in cancer' if it was decreased among the cancer cases in more than one cancer study and in at least $70 \%$ of the cancer studies that identify the feature as significant. In the same way, we defined the microbiome features as 'increased in autoimmune' and 'decreased in autoimmune'.

Data were synthesized and summarized in Microsoft Office Excel. Meta-analyses on the relative abundance of the bacterial genus were conducted in $\mathrm{R}$ version 4.0.2 [18] using $\mathrm{R}$ package metafor version 2.4-0 [19] and meta version 4.13-0 [20]. The relative abundance data were converted to log fold-change and the standard error was calculated from the $\mathrm{p}$-values, sample size and fold change using $\mathrm{R}$ package dmetar [21]. Generic inverse variance random-effect meta-analysis was performed using 'metagen' function [metagen(Log fold-change, standard error of fold-change, studlab, data $=$ data, subset $=$ genus $==$ "genus name", byvar = disease type, comb.fixed = FALSE, comb.random = TRUE, hakn = FALSE, prediction $=F A L S E$, print. byvar $\left.=F A L S E, s m={ }^{\prime \prime \prime}\right]$. The DerSimonian-Laird random effects method was used to combine the study estimates [22]. The forest plots were generated using the 'forest' function in $\mathrm{R}$ metafor package [ forest(Random effect model, ...].

\section{Results}

\section{Results of electronic database search and study selection}

We identified 3,874 potentially relevant publications by searching three electronic databases (Figure 1 , Methods). We screened the title and abstract of all publications identified in the initial database search. Primary screening identified 238 studies for full-text retrieval and eligibility checking. A total of 82 casecontrol studies were included (Supplementary Table 3-4).

\section{Characteristics and quality assessment of the included studies}

We included 37 autoimmune and 45 cancer studies, amassing 4,208 healthy human controls and 5,957 disease cases from 27 countries (Table 1, Supplementary Table 5). We captured nine types of 
autoimmune diseases: type 1 diabetes $(n=13)$ [23-35], arthritis $(n=9)$ [36-44], multiple sclerosis $(n=7)$ $[36,45-50]$, systemic lupus erythematosus $(n=4)[51-54]$, Graves' disease $(n=2)[55,56]$, primary Sjögren's syndrome $(n=1)$ [53], pemphigus vulgaris $(n=1)$ [57], anti-NMDAR encephalitis $(n=1)$ [58], and autoimmune hepatitis $(n=1)$ [59]. The cancer studies constituted ten types of cancer including colorectal cancer and adenoma $(n=27)$ [60-86], gastric cancer and adenocarcinoma $(n=6)$ [87-92], pancreatic cancer and ductal adenocarcinoma $(n=3)$ [93-95], prostate cancer $(n=2)[96,97]$, cervical cancer $(n=2)$ [98,99], lung cancer $(n=1)$ [100], thyroid papillary cancer $(n=1)$ [101], breast cancer $(n=1)$ [102], hepatocellular carcinoma $(n=1)$ [103], and GIT neoplasia $(n=1)$ [104]. Overall, the majority of the studies (79\%) have low risk of bias (based on the nine criteria of the Newcastle-Ottawa Scale) (Figure 2).

However, there was a high risk of bias in some indicators such as bias due to lack of representative cases (49\%) and improper selection of controls (44\%). The lack of representative case bias was mainly due to the absence of consecutive or obviously representative series of cases, and the improper selection of controls bias was due to inclusion of controls from hospital or no description about the selection of study control.

\section{Significant associations of alpha-diversity in cancer and autoimmune studies have opposite trends}

The majority of studies (65/82) reported alpha diversity as a measure of microbiome diversity within cases or controls (Figure 3a). The most frequently used alpha-diversity indices were Shannon, Chao and Simpson, reported in 56, 29 and 22 studies, respectively. Overall, most indices showed no significant difference in alpha-diversities between cases and controls in both cancer $(62 \%)$ and autoimmune $(67 \%)$ studies. However, an increasing trend of alpha-diversity in cancer studies and an opposite decreasing trend in autoimmune studies was observed. The overall alpha-diversity was significantly increased among cases in $25 \%$ of the cancer studies and decreased in $13 \%$. Conversely, the alpha-diversity was significantly decreased among cases in $30 \%$ of the autoimmune studies and increased in $3 \%$ (Figure $3 a$ ).

\section{Cancer and autoimmunity contain inverted and distinct microbiome signatures}

A microbiome feature can be simultaneously found to be increased in cancer and decreased in autoimmunity or vice versa. We called this type of association the "Janus-like" effect, to allude to their bidirectionality between cancer and autoimmune studies. We identified features that fall into two categories: i) cancer-increased and ii) autoimmune-increased. The 'cancer-increased' category represents the microbiome features that were found to be increased among the cancer cases but decreased or not significantly associated among the autoimmune cases. On the other hand, the 'autoimmune-increased' category represents the microbiome features that were found to be increased in autoimmune cases but decreased or not significantly associated in the cancer cases.

\section{Genus}

We identified 214 distinct genus-level associations with either cancer or autoimmune diseases across $73 / 82(89 \%)$ studies. Of these, 83 genera were reported in more than one cancer or autoimmune study. We identified 30 genera in the cancer-increased category and 10 genera in the autoimmune-increased 
category (Figure 3b). In the cancer-increased category, six genera (Enterococcus, Parabacteroides, Odoribacter, Paraprevotella, Desulfovibrio, and Oxalobacter) show true opposite directionality (i.e. increased in cancer but decreased in autoimmune diseases) between cancer and autoimmune diseases, whereas 24 genera in the cancer-increased category were increased in cancer but absent in autoimmunity. Of them, Fusobacterium, Peptostreptococcus and Porphyromonas were the most frequently increased genera among the cancer cases which were found to be associated in 13, 11 and eight cancer studies, respectively. In the autoimmune-increased category, Bacteroides and Parasutterella were inversely associated between cancer and autoimmune cases. The opposite directionality of Bacteroides between cancer and autoimmune diseases is the most prominent, which was found to be increased in 12 autoimmune studies and decreased in six cancer studies. The remaining eight genera in the autoimmune-increased category were always increased in autoimmunity but absent in cancer.

We next explored whether these associations are consistent with the relative abundance of the genus between cases and healthy controls. We extracted the relative abundance data of these significantly associated genera, where available, and conducted a random-effects meta-analysis. We conducted the meta-analysis only on genera for which we found the relative abundance data in at least four studies. By this criteria, six genera had sufficient abundance data available in at least four studies. In the autoimmune-increased genus category, meta-analysis was performed on Bacteroides and Eggerthella. Meta-analysis of Bacteroides involved 10 studies including six autoimmune and four cancer studies. The genus Bacteroides was increased among the autoimmune cases by an overall +0.24 log fold change ( $95 \% \mathrm{Cl}, 0.12$ to 0.36 ) compared with healthy controls, whereas it was decreased among the cancer cases by an overall -0.32 log fold change $(95 \% \mathrm{Cl},-0.55$ to -0.09$)$ (Figure $3 \mathrm{c})$. Between-study heterogeneity among the autoimmune and cancer studies were $48 \%$ and $66 \%$, respectively. Four autoimmune studies were included in the meta-analysis of Eggerthella, which showed an increased abundance in autoimmune cases by an overall +1.71 log fold change $(95 \% \mathrm{Cl}, 1.28$ to 2.14$)$ with a between-study heterogeneity of $0 \%$ (Figure $3 \mathrm{~d}$ ). In the cancer-increased genus category, meta-analysis was performed on Enterococcus, Parabacteroides, Peptostreptococcus and Fusobacterium. A total of eight studies were included in the meta-analysis of Enterococcus, which showed an overall increase of $+1.89 \log$ fold $(95 \% \mathrm{Cl}, 0.72$ to 3.05$)$ among the cancer cases and decrease by $-0.70 \log$ fold $(95 \% \mathrm{Cl},-1.97$ to 0.58$)$ (Figure 3e). The betweenstudy heterogeneity was $76 \%$ and $93 \%$ among the cancer and autoimmune studies, respectively. We included six studies in the meta-analysis of Parabacteroides (Figure $3 \mathrm{f}$ ). It accounted for an overall +0.93 log fold increase $(95 \% \mathrm{Cl},-1.35$ to 3.21$)$ in cancer cases and $-0.70 \log$ fold decrease $(95 \% \mathrm{Cl},-1.02$ to -0.38 ) in autoimmune cases with $82 \%$ and $19 \%$ between-study heterogeneity, respectively. Meta-analysis of Peptostreptococcus includes five cancer studies which shows an overall +3.75 log fold increase $(95 \%$ $\mathrm{Cl}, 0.90$ to 6.60 ) among the cancer cases where the between-study heterogeneity was $85 \%$ (Figure $3 \mathrm{~g}$ ). Five cancer studies were included in the meta-analysis of Fusobacterium (Figure 3h). The results showed an overall $+0.54 \log$ fold increase $(95 \% \mathrm{Cl}, 0.17$ to 0.92$)$ of Fusobacterium among the cancer cases with a between study-heterogeneity of $64 \%$.

Species 
Twenty-eight studies (34\%) reported significant species-level taxonomic associations in cancer and autoimmune diseases. A total of 159 species were significantly associated in at least one study, of which 28 species were associated in more than one cancer or autoimmune study. Finally, 20 species were identified in the cancer-increased category and seven species in the autoimmune-increased category (Figure 4a). Fusobacterium nucleatum was the most common species in the cancer-increased category, appearing in eight cancer studies. However, seven of these eight cancer studies were on colorectal cancer and one was on breast cancer. This might actually represent a skew, because colorectal cancer was the most represented cancer among the cancer studies (27/45). Therefore, it is reasonable that $F$. nucleatum would be the most common cancer-increased species and it may not indicate that $F$. nucleatum was increased in cancer generally. None of the cancer-increased species was found to be significantly associated in the autoimmune studies. Among the autoimmune-increased species, Bifidobacterium longum and Streptococcus salivarius were associated inversely between cancer and autoimmune diseases. Interestingly, four members of the Eubacterium genus, E. eligens, E. hallii, E. rectale and E. ventriosum were decreased in cancer cases in multiple studies. However, none of these Eubacterium species were significantly associated with autoimmune diseases.

\section{Metabolic pathways}

Associations between predicted microbial metabolic pathways and diseases were only reported in 20 studies (24\%). A total of 405 predicted metabolic pathways were shown to be significantly associated in at least one cancer or autoimmune study. Most of the pathways were found in only one study, whereas

48 were associated with cancer and autoimmunity in multiple studies. None of the pathways exhibited an inverse association between cancer and autoimmunity, as observed in several genera and species. However, some pathways were increased or decreased in multiple cancer and autoimmune studies such as lipopolysaccharide biosynthesis, which was found to be increased in three autoimmune studies (Figure 4b).

\section{Disease-associated genera and species are taxonomically and functionally diverse}

\section{Taxonomic diversity}

To understand the phylogenetic and taxonomic relatedness among the associated genera and species, we used the NCBI common tree taxonomy browser tool for the construction of taxonomic trees. All 40 cancer-increased and autoimmune-increased genera belonged to six bacterial phyla. The majority of the genera $(n=18)$ belonged to the phylum Firmicutes followed by Proteobacteria $(n=10)$ (Figure 5a). All 27 species were represented by the same phylum except Verrucomicrobia (Figure $5 b$ ). The Firmicutes phylum represents the majority of the species $(n=13)$ followed by Bacteroidetes $(n=6)$. We observed some interesting trends across phyla. The members (both genus and species) of Fusobacteria were always found to be increased in cancers. All the genera and species in Actinobacteria showed a positive association both in cancer and autoimmunity. Interestingly, most of the genera and species of the phylum Bacteroidetes were found to be increasingly associated with cancer and decreasingly associated with autoimmunity. The only exception is the Bacteroides genus which showed an opposite directionality 
compared with other members of this phylum (Figure 5b). Similarly, the majority of the members of the Proteobacteria showed a positive association with cancer or a negative association with autoimmunity, with the exception of the genera Parasutterella and Sutterella, which showed an opposite directionality. Most of the genera of the Firmicutes phylum were found to be increased both in cancer and autoimmunity. However, the species of the Firmicutes phylum could be divided into two groups considering opposite directionality with cancer and autoimmunity. One group of species were found to be increased in cancer ( $n=8$ species) and another group was found to be decreased in cancer $(n=5$ species) (Figure 5b).

\section{Functional diversity}

To predict the shared or unique metabolic functions among the cancer-increased or autoimmuneincreased genera and species, we identified metabolic pathways using MetAboliC pAthways DAtabase for Microbial taxonomic groups (MACADAM) [105], which employs pathway tools based on the MetaCyc database [106] that includes metabolic pathways as well as associated metabolites, reactions and enzymes. We first extracted all the metabolic pathways that are present among cancer-increased and autoimmune-increased genera and species. We then identified the pathways that were exclusively present either in cancer-increased or autoimmune-increased groups. Of the 936 metabolic pathways that were detected together in two groups, 303 pathways were found exclusively in cancer-increased taxa. On the other hand, only 17 pathways were present exclusively in autoimmune-increased taxa (Figure 5c, Supplementary file 1). Among the 303 cancer-increased pathways, 119 had a complete pathway score of 1 , meaning that some members of the cancer-increased taxa contain all enzymes to complete the metabolic pathway. The most frequent pathways in the cancer-increased groups belonged to aromatic compound degradation (38/303) followed by non-carbon nutrient metabolism (23/303), amino-acid degradation (21/303) and cofactor biosynthesis (16/303). Interestingly, 17 of the 23 non-carbon nutrient metabolism pathways were related to sulfur-metabolism. Of the autoimmune-increased pathways $(n=17)$, four complete metabolic pathways were contained in some members of the autoimmune-increased taxa. (Figure 5d, Supplementary file 1).

Because we observed taxonomically-opposing directionality within the members of Bacteroidetes, Proteobacteria and Firmicutes (Figure 5a-b), we further explored the functional similarities or dissimilarities within the members of these phyla. In the Bacteroidetes phylum, four genera and six species were associated in the cancer-increased group (Figure 5a-b), whereas only the Bacteroides genus belonged to the autoimmune-increased group. By comparing the potential metabolic functions between the cancer-increased taxa and the Bacteroides genus (autoimmune-increased) under the Bacteroidetesphylum, we detected 53 pathways exclusively present among the cancer-increased taxa and 39 pathways that were exclusively present in the genus Bacteroides (Supplementary file 1). In the Firmicutes phylum, we compared the metabolic functions between cancer-increased (i.e. Anaerococcus, Christensenella, Dialister pneumosintes, Enterococcus, Filifactor, Gemella morbillorum, Lactobacillus, Lactococcus, Megasphaera, Mogibacterium, Parvimonas, Parvimonas micra, Selenomonas, Selenomonas sputigena, and Turicibacter) and autoimmune-increased (i.e. Acidaminococcus intestini, Blautia, 
Eubacterium eligens, Eubacterium hallii, Eubacterium rectale, Flavonifractor, Gemella, Lachnoclostridium, and Streptococcus salivarius) species. A total of 162 pathways were exclusively present in the cancerincreased group, and 21 pathways were found exclusively in the autoimmune-increased group under the phylum Firmicutes (Supplementary file 1). Similarly, in the Proteobacteria phylum, we compared the metabolic functions in the cancer-increased group (i.e. Aquabacterium, Bilophila wadsworthia, Delftia, Desulfovibrio, Escherichia coli, Klebsiella, Morganella, Oxalobacter and Proteus) and autoimmuneincreased group (i.e. Sutterella). A total of 700 pathways were exclusively present in the cancer-increased group and no pathways were found exclusively in the autoimmune-increased taxa in this phylum (Supplementary file 1).

\section{Discussion}

This work involved a systematic literature review on gut microbiome studies in cancer and autoimmune conditions. We also conducted a meta-analysis to pool the relative fold-change of certain genera across multiple studies. We identified a set of microbiome features that show a consistently opposite directionality between cancer and autoimmune diseases, which we term a "Janus effect." This study also identified many non-robust microbiome associations that were only reported in a single study, illustrating the degree of variation between gut microbiome studies and the need for larger sampling or rigorous meta-analysis to distinguish significant data from noise. Most cancer studies show an increase of a group of well-known cancer associated microbiome features and decrease of commensal bacterial genera in cancer cases compared with healthy controls, whereas the autoimmune cases are characterized by invasion or depletion of commonly known commensal bacterial genera. We identified 30 genera that were categorized as cancer-increased. Of them, 24 were always increased in cancer but had no significant association with autoimmune diseases. Most of these 24 genera are previously-reported cancer-associated bacteria such as Fusobacterium, Peptostreptococcus and Porphyromonas. The genuslevel association is also consistent for some species under the same genera. Most of the 20 species found to be positively associated with cancer are cancer-associated bacteria such as Fusobacterium nucleatum [107], Peptostreptococcus stomatis [108], and Parvimonas micra [109]. These findings corroborate a previous meta-analysis of gut microbiome studies, which found increased abundance of similar microbiome features in multiple colorectal cancers studies [110]. However, six of the 30 cancerincreased genera, namely Enterococcus, Parabacteroides, Odoribacter, Paraprevotella, Desulfovibrio, and Oxalobacter show true opposite directionality between cancer and autoimmune diseases. Several species under these genera are known to be associated as potent immune suppressors. For instance, a wellknown opportunistic pathogen Enterococcus faecalis is capable of modulating immune function by suppressing macrophage activity through preventing NF-KB signaling [111] or promoting antiinflammatory cytokine IL-10 [112,113]. Similarly, members of the genus Parabacteroides including Parabacteroides distasonis were previously found to be decreased in autoimmune disease [114] and to reduce the severity of intestinal inflammation in colitis in mice through decreased production of proinflammatory cytokine TNF-a [115]. 
A set of ten genera and seven species were identified in the autoimmune-increased category. Bacteroides, Parasutterella, Bifidobacterium longum and Streptococcus salivarius were found to be inversely associated between cancer and autoimmune cases. In addition, multiple Eubacterium species were identified in this category (Figure 4). Bacteroides was found to be increased in 12 autoimmune studies and decreased in 6 cancer studies, wherase Parasutterella was found to be increased in 3 autoimmune studies and decreased in 2 cancer studies. Several species of Bacteroides and Parasutterella were previously shown to be capable of restoring anti-tumor responses such as Bacteroides thetaiotaomicron, Bacteroides rodentium, and Parasutterella excrementihominis [116-118].

Comparison of metabolic pathways across cancer-increased and autoimmune-increased microbiome taxa shows unique microbiome functions. Most noticeably, sulfur-metabolism pathways were found to be one of the frequent pathways among certain member of cancer-increased taxa such as Aquabacterium, Bilophila wadsworthia, Delftia, Desulfovibrio, Escherichia coli, Fusobacterium, Klebsiella, Lactobacillus, Morganella, Odoribacter, and Proteus. The presence of sulfur-metabolizing microbiome in the human gut has been shown to be associated with a high risk of colorectal cancer [119]. Although the exact mechanism is unknown, the intermediary sulfur-metabolites and the production of hydrogen sulfide $(\mathrm{H} 2 \mathrm{~S})$ are thought to be associated with colorectal cancer pathogenesis and epithelial damage $[120,121]$. However, disease specific causality and the underlying mechanism of these associations are yet to be discovered. Therefore, further laboratory experimentation with a combination of multiple members of these sets of microbiome features will help to understand their biological role in immunomodulation in autoimmunity and cancer.

This is the first systematic review that synthesized results from 82 case-control microbiome studies in cancer and autoimmunity, representing participants from multiple age groups and geographies. We implemented a broad and advanced search strategy in multiple databases which enable us to identify the majority of the publications in the field. We defined clear inclusion and exclusion criteria. We conducted quality assessment of the included studies using the Cochrane recommended standard tools, and maintained high standards by the strict implementation of PRISMA guidelines.

The study has some limitations. We conducted a traditional systematic review and meta-analysis rather than re-analysis of previously published sequencing data. Therefore, we mainly relied on the primary studies' sequence analysis, taxonomic classification, and statistical test. Studies typically use different models and diverse types of bioinformatics pipelines, taxonomic classifiers and statistical comparisons which may infer dissimilar taxonomies and sometimes render results difficult to compare. Re-analysis of raw sequencing data is the standard approach of performing meta-analysis of microbiome studies that provide quantitative comparison of taxonomies across diseases. However, there are also many challenges of a meta-analysis of raw sequencing data such as diverse study design, heterogeneous demographics of study participants, different methods for DNA extraction and sequencing type and platform, and availability of metadata and raw sequences. For instance, in our study, many of the included studies $(>50 \%)$ do not have publicly available raw sequencing data. Moreover, the findings of this study are very similar to a previous meta-analysis [110] where the authors performed re-analysis of 
raw sequencing data of 28 published case-control studies across ten diseases. They detected an increased abundance of common cancer-associated bacteria such as Fusobacterium, Porphyromonas, Peptostreptococcus, and Parvimonas in multiple colorectal cancer studies. We found similar associations for all of these bacteria in multiple cancer studies in our analysis. Finally, we were not able to conduct a meta-analysis on many genera and species due to the absence of relative abundance data in the published literature.

Based on our findings and observations, we propose some recommendations and future directions in this field of research. We recommend that future case-control microbiome studies should report relative abundance data, sample variance and $p$-value of the statistical analysis which will facilitate future metaanalysis and enable combining of results from multiple studies. The importance of publicly available raw sequencing data and patient metadata is enormous for direct comparison of the results across studies. The findings from this study can be used to identify selective microbial consortia for microbiome-based immune therapies for the prevention and treatment of cancer and autoimmune diseases which can be further tested by laboratory investigation.

In conclusion, despite some limitations, this study identified distinct types of cancer and autoimmune associated microbiome features that are consistently identified in multiple studies. These associations may point to important underlying biology of how disease and health shape the microbiome or vice versa, and this serves as an unprecedented trove of hypotheses for future studies of the role of the microbiome in autoimmunity and cancer.

\section{Abbreviations}

EMBAS: Excerpta Medica dataBASE

PRISMA: Preferred Reporting Items for Systematic Reviews and Meta-Analyses

FDR: False discovery rate

GIT: Gastrointestinal tract

H2S: Hydrogen sulfide

MACADAM: MetAboliC pAthways DAtabase for Microbial taxonomic groups

MeSH: Medical Subject Headings

NMDAR: Anti-N-methyl-D-aspartate receptor

NOS: Newcastle-Ottawa Scale

T1D: Type 1 diabetes 


\section{Declarations}

\section{Ethical Approval and Consent to participate}

This study uses data from publicly available scientific literature and does not involve any human or animal subject. Therefore, ethics approval is not required for this study.

\section{Consent for publication}

Not applicable.

\section{Availability of data and materials}

All data that support the results of this study are available from the corresponding author on request. We included a complete list of all included and excluded studies as supplementary files.

\section{Competing interests}

Not applicable.

\section{Funding}

The salary of the author MZI was supported by Lundbeck Foundation, Copenhagen, Denmark (Grant ID R288-2018-1123).

\section{Author contributions}

Conceptualization: MZI, TX, ADK; Database search: MZI; Article screening and data extraction: MZI, MT; Bioinformatic and statistical analysis: MZI, BTT, TX, CP, ADK; First manuscript draft: MZI; Manuscript revision: MZI, MT, TX, BTT, CP, ADK.

\section{Acknowledgments}

MZI acknowledges support for postdoctoral fellowships from the Lundbeck Foundation, Copenhagen, Denmark.

\section{Authors' information}

Section on Pathophysiology and Molecular Pharmacology, Joslin Diabetes Center, Boston, Massachusetts, United States of America

Md Zohorul Islam, Melissa Tran, Tao Xu, Braden T. Tierney, Chirag Patel, Aleksandar David Kostic Department of Microbiology, Harvard Medical School, Boston, Massachusetts, United States of America Md Zohorul Islam, Melissa Tran, Tao Xu, Braden T. Tierney, Aleksandar David Kostic 
Department of Veterinary and Animal Sciences, University of Copenhagen, Denmark

Md Zohorul Islam

Department of Biomedical Informatics, Harvard Medical School, Boston, Massachusetts, United States of America

Chirag Patel

Section on Islet Cell and Regenerative Biology, Joslin Diabetes Center, Boston, Massachusetts, United States of America

Aleksandar David Kostic

Corresponding author:Aleksandar David Kostic, email: aleksandar.kostic@joslin.harvard.edu

\section{References}

1. Dunn GP, Old LJ, Schreiber RD. The immunobiology of cancer immunosurveillance and immunoediting. Immunity. 2004;21:137-48.

2. Rosenblum MD, Remedios KA, Abbas AK. Mechanisms of human autoimmunity. J Clin Invest. 2015;125:2228-33.

3. Joshi NS, Akama-Garren EH, Lu Y, Lee D-Y, Chang GP, Li A, et al. Regulatory T Cells in TumorAssociated Tertiary Lymphoid Structures Suppress Anti-tumor T Cell Responses. Immunity. 2015;43:579-90.

4. Allaoui R, Hagerling C, Desmond E, Warfvinge C-F, Jirström K, Leandersson K. Infiltration of $y \delta T$ cells, IL-17+ T cells and FoxP3+ T cells in human breast cancer. Cancer Biomark. 2017;20:395-409.

5. Togashi Y, Shitara K, Nishikawa H. Regulatory T cells in cancer immunosuppression - implications for anticancer therapy [Internet]. Nature Reviews Clinical Oncology. 2019. p. 356-71. Available from: http://dx.doi.org/10.1038/s41571-019-0175-7

6. Szypowska A, Stelmaszczyk-Emmel A, Demkow U, Luczyński W. Low frequency of regulatory T cells in the peripheral blood of children with type 1 diabetes diagnosed under the age of five. Arch Immunol Ther Exp . 2012;60:307-13.

7. Zhang X, Zhang X, Zhuang L, Xu C, Li T, Zhang G, et al. Decreased regulatory T-cell frequency and interleukin-35 levels in patients with rheumatoid arthritis. Exp Ther Med. 2018;16:5366-72.

8. Liu M-F, Wang C-R, Fung L-L, Wu C-R. Decreased CD4+CD25+ T cells in peripheral blood of patients with systemic lupus erythematosus. Scand J Immunol. 2004;59:198-202.

9. Crispin JC, Martínez A, Alcocer-Varela J. Quantification of regulatory T cells in patients with systemic lupus erythematosus. J Autoimmun. 2003;21:273-6.

10. Barreto M, Ferreira RC, Lourenço L, Moraes-Fontes MF, Santos E, Alves M, et al. Low frequency of CD4+CD25+ Treg in SLE patients: a heritable trait associated with CTLA4 and TGFbeta gene 
variants. BMC Immunol. 2009;10:5.

11. Grant CR, Liberal R, Mieli-Vergani G, Vergani D, Longhi MS. Regulatory T-cells in autoimmune diseases: Challenges, controversies and-yet-unanswered questions. Autoimmun Rev. 2015;14:105-16.

12. Schwabe RF, Jobin C. The microbiome and cancer. Nat Rev Cancer. 2013;13:800-12.

13. Zhang X, Chen B-D, Zhao L-D, Li H. The Gut Microbiota: Emerging Evidence in Autoimmune Diseases. Trends Mol Med. 2020;26:862-73.

14. Shi Y, Mu L. An expanding stage for commensal microbes in host immune regulation. Cell Mol Immunol. 2017;14:339-48.

15. Macpherson AJ, Harris NL. Interactions between commensal intestinal bacteria and the immune system. Nat Rev Immunol. 2004;4:478-85.

16. Moher D, Liberati A, Tetzlaff J, Altman DG, PRISMA Group. Preferred reporting items for systematic reviews and meta-analyses: the PRISMA statement. PLoS Med. 2009;6:e1000097.

17. Wells G, Shea B, O'Connell D, Peterson J, Welch V, Losos M, et al. The Newcastle-Ottawa Scale (NOS) for assessing the quality of nonrandomised studies in meta-analyses. Ottawa, Canada: Ottawa Hospital Research Institute. 2013.

18. Team RC. R: A language and environment for statistical com-puting. Vienna, Austria: R Foundation for Statistical Computing. Retrieved from ...; 2017.

19. Viechtbauer W. Conducting meta-analyses in R with the metafor package. J Stat Softw. UCLA Statistics; 2010;36:1-48.

20. Schwarzer G, Carpenter JR, Rücker G. Meta-Analysis with R. Springer; 2015.

21. Harrer M, Cuijpers P, Furukawa T, Ebert DD. dmetar: Companion R Package For The Guide “Doing Meta-Analysis in R". R package version 0.0. 9000. 2019.

22. DerSimonian R, Laird N. Meta-analysis in clinical trials. Control Clin Trials. 1986;7:177-88.

23. Harbison JE, Roth-Schulze AJ, Giles LC, Tran CD, Ngui KM, Penno MA, et al. Gut microbiome dysbiosis and increased intestinal permeability in children with islet autoimmunity and type 1 diabetes: A prospective cohort study. Pediatr Diabetes. 2019;20:574-83.

24. Vatanen T, Franzosa EA, Schwager R, Tripathi S, Arthur TD, Vehik K, et al. The human gut microbiome in early-onset type 1 diabetes from the TEDDY study. Nature. 2018;562:589-94.

25. Stewart CJ, Ajami NJ, O’Brien JL, Hutchinson DS, Smith DP, Wong MC, et al. Temporal development of the gut microbiome in early childhood from the TEDDY study. Nature. 2018;562:583-8.

26. Leiva-Gea I, Sánchez-Alcoholado L, Martín-Tejedor B, Castellano-Castillo D, Moreno-Indias I, UrdaCardona A, et al. Gut Microbiota Differs in Composition and Functionality Between Children With Type 1 Diabetes and MODY2 and Healthy Control Subjects: A Case-Control Study. Diabetes Care. 2018;41:2385-95.

27. Cinek O, Kramna L, Mazankova K, Odeh R, Alassaf A, Ibekwe MU, et al. The bacteriome at the onset of type 1 diabetes: A study from four geographically distant African and Asian countries. Diabetes 
Res Clin Pract. 2018;144:51-62.

28. de Groot PF, Belzer C, Aydin Ö, Levin E, Levels JH, Aalvink S, et al. Distinct fecal and oral microbiota composition in human type 1 diabetes, an observational study. PLoS One. 2017;12:e0188475.

29. Singh H, Yu Y, Suh M-J, Torralba MG, Stenzel RD, Tovchigrechko A, et al. Type 1 Diabetes: Urinary Proteomics and Protein Network Analysis Support Perturbation of Lysosomal Function.

Theranostics. 2017;7:2704-17.

30. Qi C-J, Zhang Q, Yu M, Xu J-P, Zheng J, Wang T, et al. Imbalance of Fecal Microbiota at Newly Diagnosed Type 1 Diabetes in Chinese Children. Chin Med J . 2016;129:1298-304.

31. Stewart CJ, Nelson A, Campbell MD, Walker M, Stevenson EJ, Shaw JA, et al. Gut microbiota of Type 1 diabetes patients with good glycaemic control and high physical fitness is similar to people without diabetes: an observational study. Diabet Med. 2017;34:127-34.

32. Alkanani AK, Hara N, Gottlieb PA, Ir D, Robertson CE, Wagner BD, et al. Alterations in Intestinal Microbiota Correlate With Susceptibility to Type 1 Diabetes. Diabetes. 2015;64:3510-20.

33. Mejía-León ME, Petrosino JF, Ajami NJ, Domínguez-Bello MG, de la Barca AMC. Fecal microbiota imbalance in Mexican children with type 1 diabetes. Sci Rep. 2014;4:3814.

34. Huang Y, Li S-C, Hu J, Ruan H-B, Guo H-M, Zhang H-H, et al. Gut microbiota profiling in Han Chinese with type 1 diabetes. Diabetes Res Clin Pract. 2018;141:256-63.

35. Salamon D, Sroka-Oleksiak A, Kapusta P, Szopa M, Mrozińska S, Ludwig-Słomczyńska AH, et al. Characteristics of gut microbiota in adult patients with type 1 and type 2 diabetes based on next-generation sequencing of the 16S rRNA gene fragment. Pol Arch Intern Med. 2018;128:336-43.

36. Forbes JD, Chen C-Y, Knox NC, Marrie R-A, El-Gabalawy H, de Kievit T, et al. A comparative study of the gut microbiota in immune-mediated inflammatory diseases-does a common dysbiosis exist? Microbiome. 2018;6:221.

37. Picchianti-Diamanti A, Panebianco C, Salemi S, Sorgi ML, Di Rosa R, Tropea A, et al. Analysis of Gut Microbiota in Rheumatoid Arthritis Patients: Disease-Related Dysbiosis and Modifications Induced by Etanercept. Int J Mol Sci [Internet]. 2018;19. Available from: http://dx.doi.org/10.3390/ijms19102938

38. Wen C, Zheng Z, Shao T, Liu L, Xie Z, Le Chatelier E, et al. Quantitative metagenomics reveals unique gut microbiome biomarkers in ankylosing spondylitis. Genome Biol. 2017;18:142.

39. Breban M, Tap J, Leboime A, Said-Nahal R, Langella P, Chiocchia G, et al. Faecal microbiota study reveals specific dysbiosis in spondyloarthritis. Ann Rheum Dis. 2017;76:1614-22.

40. Aggarwal A, Sarangi AN, Gaur P, Shukla A, Aggarwal R. Gut microbiome in children with enthesitisrelated arthritis in a developing country and the effect of probiotic administration. Clin Exp Immunol. 2017;187:480-9.

41. Chen J, Wright K, Davis JM, Jeraldo P, Marietta EV, Murray J, et al. An expansion of rare lineage intestinal microbes characterizes rheumatoid arthritis. Genome Med. 2016;8:43. 
42. Tejesvi MV, Arvonen M, Kangas SM, Keskitalo PL, Pirttilä AM, Karttunen TJ, et al. Faecal microbiome in new-onset juvenile idiopathic arthritis. Eur J Clin Microbiol Infect Dis. 2016;35:363-70.

43. Stoll ML, Kumar R, Morrow CD, Lefkowitz EJ, Cui X, Genin A, et al. Altered microbiota associated with abnormal humoral immune responses to commensal organisms in enthesitis-related arthritis. Arthritis Res Ther. 2014;16:486.

44. Sun Y, Chen Q, Lin P, Xu R, He D, Ji W, et al. Characteristics of Gut Microbiota in Patients With Rheumatoid Arthritis in Shanghai, China. Front Cell Infect Microbiol. 2019;9:369.

45. Jangi S, Gandhi R, Cox LM, Li N, von Glehn F, Yan R, et al. Alterations of the human gut microbiome in multiple sclerosis. Nat Commun. 2016;7:12015.

46. Chen J, Chia N, Kalari KR, Yao JZ, Novotna M, Paz Soldan MM, et al. Multiple sclerosis patients have a distinct gut microbiota compared to healthy controls. Sci Rep. 2016;6:28484.

47. Miyake S, Kim S, Suda W, Oshima K, Nakamura M, Matsuoka T, et al. Dysbiosis in the Gut Microbiota of Patients with Multiple Sclerosis, with a Striking Depletion of Species Belonging to Clostridia XIVa and IV Clusters. PLoS One. 2015;10:e0137429.

48. Reynders T, Devolder L, Valles-Colomer M, Van Remoortel A, Joossens M, De Keyser J, et al. Gut microbiome variation is associated to Multiple Sclerosis phenotypic subtypes. Ann Clin Transl Neurol. 2020;7:406-19.

49. Choileáin SN, Kleinewietfeld M, Raddassi K, Hafler DA, Ruff WE, Longbrake EE. CXCR3+ T cells in multiple sclerosis correlate with reduced diversity of the gut microbiome. J Transl Autoimmun. 2020;3:100032.

50. Tremlett H, Fadrosh DW, Faruqi AA, Zhu F, Hart J, Roalstad S, et al. Gut microbiota in early pediatric multiple sclerosis: a case-control study. Eur J Neurol. 2016;23:1308-21.

51. Wei F, Xu H, Yan C, Rong C, Liu B, Zhou H. Changes of intestinal flora in patients with systemic lupus erythematosus in northeast China. PLoS One. 2019;14:e0213063.

52. Hevia A, Milani C, López P, Cuervo A, Arboleya S, Duranti S, et al. Intestinal dysbiosis associated with systemic lupus erythematosus. MBio. 2014;5:e01548-14.

53. van der Meulen TA, Harmsen HJM, Vila AV, Kurilshikov A, Liefers SC, Zhernakova A, et al. Shared gut, but distinct oral microbiota composition in primary Sjögren's syndrome and systemic lupus erythematosus. J Autoimmun. 2019;97:77-87.

54. Zhang S-X, Wang J, Chen J-W, Zhang M-X, Zhang Y-F, Hu F-Y, et al. The level of peripheral regulatory $T$ cells is linked to changes in gut commensal microflora in patients with systemic lupus erythematosus. Ann Rheum Dis [Internet]. 2019; Available from:

http://dx.doi.org/10.1136/annrheumdis-2019-216504

55. Ishaq HM, Mohammad IS, Shahzad M, Ma C, Raza MA, Wu X, et al. Molecular Alteration Analysis of Human Gut Microbial Composition in Graves' disease Patients. Int J Biol Sci. 2018;14:1558-70.

56. Shi T-T, Xin Z, Hua L, Zhao R-X, Yang Y-L, Wang H, et al. Alterations in the intestinal microbiota of patients with severe and active Graves' orbitopathy: a cross-sectional study [Internet]. Journal of 
Endocrinological Investigation. 2019. p. 967-78. Available from: http://dx.doi.org/10.1007/s40618019-1010-9

57. Huang S, Mao J, Zhou L, Xiong X, Deng Y. The imbalance of gut microbiota and its correlation with plasma inflammatory cytokines in pemphigus vulgaris patients. Scand J Immunol. 2019;90:e12799.

58. Gong X, Liu X, Li C, Chen C, Lin J, Li A, et al. Alterations in the human gut microbiome in anti-Nmethyl-D-aspartate receptor encephalitis. Ann Clin Transl Neurol. 2019;6:1771-81.

59. Wei Y, Li Y, Yan L, Sun C, Miao Q, Wang Q, et al. Alterations of gut microbiome in autoimmune hepatitis. Gut. 2020;69:569-77.

60. Yachida S, Mizutani S, Shiroma H, Shiba S, Nakajima T, Sakamoto T, et al. Metagenomic and metabolomic analyses reveal distinct stage-specific phenotypes of the gut microbiota in colorectal cancer. Nat Med. 2019;25:968-76.

61. Sarhadi V, Lahti L, Saberi F, Youssef O, Kokkola A, Karla T, et al. Gut Microbiota and Host Gene Mutations in Colorectal Cancer Patients and Controls of Iranian and Finnish Origin [Internet]. Anticancer Research. 2020. p. 1325-34. Available from: http://dx.doi.org/10.21873/anticanres.14074

62. Saito K, Koido S, Odamaki T, Kajihara M, Kato K, Horiuchi S, et al. Metagenomic analyses of the gut microbiota associated with colorectal adenoma. PLoS One. 2019;14:e0212406.

63. Hannigan GD, Duhaime MB, Ruffin MT 4th, Koumpouras CC, Schloss PD. Diagnostic Potential and Interactive Dynamics of the Colorectal Cancer Virome. MBio [Internet]. 2018;9. Available from: http://dx.doi.org/10.1128/mBio.02248-18

64. Zhang Y, Yu X, Yu E, Wang N, Cai Q, Shuai Q, et al. Changes in gut microbiota and plasma inflammatory factors across the stages of colorectal tumorigenesis: a case-control study. BMC Microbiol. 2018;18:92.

65. Allali I, Boukhatem N, Bouguenouch L, Hardi H, Boudouaya HA, Cadenas MB, et al. Gut microbiome of Moroccan colorectal cancer patients. Med Microbiol Immunol. 2018;207:211-25.

66. Vogtmann E, Hua X, Zeller G, Sunagawa S, Voigt AY, Hercog R, et al. Colorectal Cancer and the Human Gut Microbiome: Reproducibility with Whole-Genome Shotgun Sequencing. PLoS One. 2016;11:e0155362.

67. Kasai C, Sugimoto K, Moritani I, Tanaka J, Oya Y, Inoue H, et al. Comparison of human gut microbiota in control subjects and patients with colorectal carcinoma in adenoma: Terminal restriction fragment length polymorphism and next-generation sequencing analyses. Oncol Rep. 2016;35:325-33.

68. Nakatsu G, Li X, Zhou H, Sheng J, Wong SH, Wu WKK, et al. Gut mucosal microbiome across stages of colorectal carcinogenesis. Nat Commun. 2015;6:8727.

69. Feng Q, Liang S, Jia H, Stadlmayr A, Tang L, Lan Z, et al. Gut microbiome development along the colorectal adenoma-carcinoma sequence. Nat Commun. 2015;6:6528.

70. Zeller G, Tap J, Voigt AY, Sunagawa S, Kultima JR, Costea PI, et al. Potential of fecal microbiota for early-stage detection of colorectal cancer. Mol Syst Biol. 2014;10:766. 
71. Zackular JP, Rogers MAM, Ruffin MT 4th, Schloss PD. The human gut microbiome as a screening tool for colorectal cancer. Cancer Prev Res . 2014;7:1112-21.

72. Ahn J, Sinha R, Pei Z, Dominianni C, Wu J, Shi J, et al. Human gut microbiome and risk for colorectal cancer. J Natl Cancer Inst. 2013;105:1907-11.

73. Wu N, Yang X, Zhang R, Li J, Xiao X, Hu Y, et al. Dysbiosis signature of fecal microbiota in colorectal cancer patients. Microb Ecol. 2013;66:462-70.

74. Chen H-M, Yu Y-N, Wang J-L, Lin Y-W, Kong X, Yang C-Q, et al. Decreased dietary fiber intake and structural alteration of gut microbiota in patients with advanced colorectal adenoma. Am J Clin Nutr. 2013;97:1044-52.

75. Sanapareddy N, Legge RM, Jovov B, McCoy A, Burcal L, Araujo-Perez F, et al. Increased rectal microbial richness is associated with the presence of colorectal adenomas in humans. ISME J. 2012;6:1858-68.

76. Wang T, Cai G, Qiu Y, Fei N, Zhang M, Pang X, et al. Structural segregation of gut microbiota between colorectal cancer patients and healthy volunteers. ISME J. 2012;6:320-9.

77. Sheng Q, Du H, Cheng X, Cheng X, Tang Y, Pan L, et al. Characteristics of fecal gut microbiota in patients with colorectal cancer at different stages and different sites. Oncol Lett. 2019;18:4834-44.

78. Tarallo S, Ferrero G, Gallo G, Francavilla A, Clerico G, Realis Luc A, et al. Altered Fecal Small RNA Profiles in Colorectal Cancer Reflect Gut Microbiome Composition in Stool Samples. mSystems [Internet]. 2019;4. Available from: http://dx.doi.org/10.1128/mSystems.00289-19

79. Yang T-W, Lee W-H, Tu S-J, Huang W-C, Chen H-M, Sun T-H, et al. Enterotype-based Analysis of Gut Microbiota along the Conventional Adenoma-Carcinoma Colorectal Cancer Pathway. Sci Rep. 2019;9:10923.

80. Yang Y, Misra BB, Liang L, Bi D, Weng W, Wu W, et al. Integrated microbiome and metabolome analysis reveals a novel interplay between commensal bacteria and metabolites in colorectal cancer. Theranostics. 2019;9:4101-14.

81. Gao Z, Guo B, Gao R, Zhu Q, Qin H. Microbiota disbiosis is associated with colorectal cancer. Front Microbiol. 2015;6:20.

82. Chen W, Liu F, Ling Z, Tong X, Xiang C. Human intestinal lumen and mucosa-associated microbiota in patients with colorectal cancer. PLoS One. 2012;7:e39743.

83. Hale VL, Chen J, Johnson S, Harrington SC, Yab TC, Smyrk TC, et al. Shifts in the Fecal Microbiota Associated with Adenomatous Polyps. Cancer Epidemiol Biomarkers Prev. 2017;26:85-94.

84. Mira-Pascual L, Cabrera-Rubio R, Ocon S, Costales P, Parra A, Suarez A, et al. Microbial mucosal colonic shifts associated with the development of colorectal cancer reveal the presence of different bacterial and archaeal biomarkers. J Gastroenterol. 2015;50:167-79.

85. Weir TL, Manter DK, Sheflin AM, Barnett BA, Heuberger AL, Ryan EP. Stool microbiome and metabolome differences between colorectal cancer patients and healthy adults. PLoS One. 2013;8:e70803. 
86. Yu J, Feng Q, Wong SH, Zhang D, Liang QY, Qin Y, et al. Metagenomic analysis of faecal microbiome as a tool towards targeted non-invasive biomarkers for colorectal cancer. Gut. 2017;66:70-8.

87. Liang $W$, Yang $Y$, Wang $H$, Wang $H, Y u X$, Lu Y, et al. Gut microbiota shifts in patients with gastric cancer in perioperative period. Medicine . 2019;98:e16626.

88. Qi Y-F, Sun J-N, Ren L-F, Cao X-L, Dong J-H, Tao K, et al. Intestinal Microbiota Is Altered in Patients with Gastric Cancer from Shanxi Province, China. Dig Dis Sci. 2019;64:1193-203.

89. Castaño-Rodríguez N, Goh K-L, Fock KM, Mitchell HM, Kaakoush NO. Dysbiosis of the microbiome in gastric carcinogenesis. Sci Rep. 2017;7:15957.

90. Jo HJ, Kim J, Kim N, Park JH, Nam RH, Seok Y-J, et al. Analysis of Gastric Microbiota by Pyrosequencing: Minor Role of Bacteria Other Than Helicobacter pylori in the Gastric Carcinogenesis. Helicobacter. 2016;21:364-74.

91. Gunathilake MN, Lee J, Choi IJ, Kim Y-I, Ahn Y, Park C, et al. Association between the relative abundance of gastric microbiota and the risk of gastric cancer: a case-control study. Sci Rep. 2019;9:13589.

92. Gantuya B, El Serag HB, Matsumoto T, Ajami NJ, Uchida T, Oyuntsetseg K, et al. Gastric mucosal microbiota in a Mongolian population with gastric cancer and precursor conditions [Internet]. Alimentary Pharmacology \& Therapeutics. 2020. p. 770-80. Available from: http://dx.doi.org/10.1111/apt.15675

93. Mei Q-X, Huang C-L, Luo S-Z, Zhang X-M, Zeng Y, Lu Y-Y. Characterization of the duodenal bacterial microbiota in patients with pancreatic head cancer vs. healthy controls. Pancreatology. 2018;18:438-45.

94. Pushalkar S, Hundeyin M, Daley D, Zambirinis CP, Kurz E, Mishra A, et al. The Pancreatic Cancer Microbiome Promotes Oncogenesis by Induction of Innate and Adaptive Immune Suppression. Cancer Discov. 2018;8:403-16.

95. Half E, Keren N, Reshef L, Dorfman T, Lachter I, Kluger Y, et al. Fecal microbiome signatures of pancreatic cancer patients. Sci Rep. 2019;9:16801.

96. Alanee S, El-Zawahry A, Dynda D, Dabaja A, McVary K, Karr M, et al. A prospective study to examine the association of the urinary and fecal microbiota with prostate cancer diagnosis after transrectal biopsy of the prostate using 16sRNA gene analysis. Prostate. 2019;79:81-7.

97. Golombos DM, Ayangbesan A, O'Malley P, Lewicki P, Barlow L, Barbieri CE, et al. The Role of Gut Microbiome in the Pathogenesis of Prostate Cancer: A Prospective, Pilot Study. Urology. 2018;111:122-8.

98. Sims TT, Colbert LE, Zheng J, Delgado Medrano AY, Hoffman KL, Ramondetta L, et al. Gut microbial diversity and genus-level differences identified in cervical cancer patients versus healthy controls. Gynecol Oncol. 2019;155:237-44.

99. Wang Z, Wang Q, Zhao J, Gong L, Zhang Y, Wang X, et al. Altered diversity and composition of the gut microbiome in patients with cervical cancer. AMB Express. 2019;9:40. 
100. Zhuang $\mathrm{H}$, Cheng L, Wang Y, Zhang Y-K, Zhao M-F, Liang G-D, et al. Dysbiosis of the Gut Microbiome in Lung Cancer. Front Cell Infect Microbiol. 2019;9:112.

101. Feng J, Zhao F, Sun J, Lin B, Zhao L, Liu Y, et al. Alterations in the gut microbiota and metabolite profiles of thyroid carcinoma patients. Int J Cancer. 2019;144:2728-45.

102. Zhu J, Liao M, Yao Z, Liang W, Li Q, Liu J, et al. Breast cancer in postmenopausal women is associated with an altered gut metagenome. Microbiome. 2018;6:136.

103. Ren Z, Li A, Jiang J, Zhou L, Yu Z, Lu H, et al. Gut microbiome analysis as a tool towards targeted non-invasive biomarkers for early hepatocellular carcinoma. Gut. 2019;68:1014-23.

104. Youssef O, Lahti L, Kokkola A, Karla T, Tikkanen M, Ehsan H, et al. Stool Microbiota Composition Differs in Patients with Stomach, Colon, and Rectal Neoplasms. Dig Dis Sci. 2018;63:2950-8.

105. Le Boulch M, Déhais P, Combes S, Pascal G. The MACADAM database: a MetAboliC pAthways DAtabase for Microbial taxonomic groups for mining potential metabolic capacities of archaeal and bacterial taxonomic groups. Database [Internet]. Oxford Academic; 2019 [cited 2020 Oct 21];2019. Available from: https://academic.oup.com/database/articleabstract/doi/10.1093/database/baz049/5480179

106. Caspi R, Billington R, Keseler IM, Kothari A, Krummenacker M, Midford PE, et al. The MetaCyc database of metabolic pathways and enzymes - a 2019 update. Nucleic Acids Res. 2020;48:D44553.

107. Brennan CA, Garrett WS. Fusobacterium nucleatum - symbiont, opportunist and oncobacterium. Nat Rev Microbiol. 2019;17:156-66.

108. Long X, Wong CC, Tong L, Chu ESH, Ho Szeto C, Go MYY, et al. Peptostreptococcus anaerobius promotes colorectal carcinogenesis and modulates tumour immunity. Nat Microbiol. 2019;4:231930 .

109. Löwenmark T, Löfgren-Burström A, Zingmark C, Eklöf V, Dahlberg M, Wai SN, et al. Parvimonas micra as a putative non-invasive faecal biomarker for colorectal cancer. Sci Rep. 2020;10:15250.

110. Duvallet C, Gibbons SM, Gurry T, Irizarry RA, Alm EJ. Meta-analysis of gut microbiome studies identifies disease-specific and shared responses. Nat Commun. 2017;8:1784.

111. Tien BYQ, Goh HMS, Chong KKL, Bhaduri-Tagore S, Holec S, Dress R, et al. Enterococcus faecalis Promotes Innate Immune Suppression and Polymicrobial Catheter-Associated Urinary Tract Infection. Infect Immun [Internet]. 2017;85. Available from: http://dx.doi.org/10.1128/IAl.00378-17

112. Takada Y, Hisamatsu T, Kamada N, Kitazume MT, Honda H, Oshima Y, et al. Monocyte Chemoattractant Protein-1 Contributes to Gut Homeostasis and Intestinal Inflammation by Composition of IL-10-Producing Regulatory Macrophage Subset [Internet]. The Journal of Immunology. 2010. p. 2671-6. Available from: http://dx.doi.org/10.4049/jimmunol.0804012

113. Kao PHN, Kline KA. Dr. Jekyll and Mr. Hide: How Enterococcus faecalis Subverts the Host Immune Response to Cause Infection. J Mol Biol. 2019;431:2932-45.

114. Cekanaviciute E, Yoo BB, Runia TF, Debelius JW, Singh S, Nelson CA, et al. Gut bacteria from multiple sclerosis patients modulate human T cells and exacerbate symptoms in mouse models. Proc Natl 
Acad Sci U S A. 2017;114:10713-8.

115. Kverka M, Zakostelska Z, Klimesova K, Sokol D, Hudcovic T, Hrncir T, et al. Oral administration of Parabacteroides distasonis antigens attenuates experimental murine colitis through modulation of immunity and microbiota composition. Clin Exp Immunol. 2011;163:250-9.

116. Li Y, Elmén L, Segota I, Xian Y, Tinoco R, Feng Y, et al. Prebiotic-Induced Anti-tumor Immunity Attenuates Tumor Growth. Cell Rep. 2020;30:1753-66.e6.

117. Vétizou M, Pitt JM, Daillère R, Lepage $P$, Waldschmitt $N$, Flament $C$, et al. Anticancer immunotherapy by CTLA-4 blockade relies on the gut microbiota. Science. American Association for the Advancement of Science; 2015;350:1079-84.

118. Li Y, Tinoco R, Elmén L, Segota I, Xian Y, Fujita Y, et al. Gut microbiota dependent anti-tumor immunity restricts melanoma growth in Rnf5-/- mice. Nat Commun. Nature Publishing Group; 2019;10:1-16.

119. Nguyen LH, Ma W, Wang DD, Cao Y, Mallick H, Gerbaba TK, et al. Association Between SulfurMetabolizing Bacterial Communities in Stool and Risk of Distal Colorectal Cancer in Men. Gastroenterology. 2020;158:1313-25.

120. Attene-Ramos MS, Wagner ED, Gaskins HR, Plewa MJ. Hydrogen Sulfide Induces Direct RadicalAssociated DNA Damage [Internet]. Molecular Cancer Research. 2007. p. 455-9. Available from: http://dx.doi.org/10.1158/1541-7786.mcr-06-0439

121. Cai W-J, Wang M-J, Ju L-H, Wang C, Zhu Y-C. Hydrogen sulfide induces human colon cancer cell proliferation: role of Akt, ERK and p21. Cell Biol Int. 2010;34:565-72.

\section{Tables}

Table 1. Summary of study characteristics. 


\begin{tabular}{|c|c|c|c|c|c|c|c|}
\hline Categories & Disease & $\begin{array}{l}\text { Country } \\
\text { (ISO2) }\end{array}$ & $\begin{array}{l}\text { No. } \\
\text { study }\end{array}$ & $\begin{array}{l}\text { No. } \\
\text { case }\end{array}$ & $\begin{array}{l}\text { No. } \\
\text { control }\end{array}$ & $\begin{array}{l}\text { Age- } \\
\text { range } \\
\text { (years) }\end{array}$ & $\begin{array}{l}\text { Sequencing } \\
\text { type (no. } \\
\text { study) }\end{array}$ \\
\hline \multirow[t]{9}{*}{ Autoimmune } & Type 1 diabetes & $\begin{array}{l}\text { AU, AZ, CN, } \\
\text { FI, DE, JO, } \\
\text { MX, NG, } \\
\text { PL, ES, SD, } \\
\text { SE, NL, UK, } \\
\text { US }\end{array}$ & 13 & 568 & 893 & $1-47$ & $\begin{array}{l}16 s(12) \\
\text { and } \\
\text { shotgun (1) }\end{array}$ \\
\hline & Arthritis* & $\begin{array}{l}\text { CA, CN, FI, } \\
\text { FR, IN, IT, } \\
\text { US }\end{array}$ & 9 & 438 & 339 & $5-71$ & $\begin{array}{l}16 s(8) \text { and } \\
\text { shotgun (1) }\end{array}$ \\
\hline & $\begin{array}{l}\text { Multiple } \\
\text { sclerosis }\end{array}$ & $\begin{array}{l}\text { CN, BE, JP, } \\
\text { US }\end{array}$ & 7 & 272 & 328 & $12-50$ & $16 s$ \\
\hline & $\begin{array}{l}\text { Systemic lupus } \\
\text { erythematosus }\end{array}$ & $\mathrm{CN}, \mathrm{ES}, \mathrm{NL}$ & 4 & 156 & 253 & $45-50$ & $16 s$ \\
\hline & Graves' Disease & $\mathrm{CN}$ & 2 & 43 & 42 & $35-50$ & $16 s$ \\
\hline & $\begin{array}{l}\text { Primary } \\
\text { Sjögren's } \\
\text { syndrome }\end{array}$ & $\mathrm{NL}$ & 1 & 39 & 965 & $40-55$ & $16 s$ \\
\hline & $\begin{array}{l}\text { Pemphigus } \\
\text { vulgaris }\end{array}$ & $\mathrm{CN}$ & 1 & 18 & 14 & $44-45$ & $16 s$ \\
\hline & $\begin{array}{l}\text { Anti-NMDAR } \\
\text { encephalitis }\end{array}$ & $\mathrm{CN}$ & 1 & 30 & 12 & $29-32$ & $16 s$ \\
\hline & $\begin{array}{l}\text { Autoimmune } \\
\text { hepatitis }\end{array}$ & $\mathrm{CN}$ & 1 & 119 & 132 & $19-75$ & $16 s$ \\
\hline \multirow[t]{7}{*}{ Cancer } & $\begin{array}{l}\text { Colorectal } \\
\text { cancer and } \\
\text { adenoma }\end{array}$ & $\begin{array}{l}\text { AT, CA, CN, } \\
\text { DK, FI, FR, } \\
\text { IR, IT, JP, } \\
\text { MA, ES, US }\end{array}$ & 27 & 1630 & 2131 & $40-70$ & $\begin{array}{l}16 s(22) \\
\text { and } \\
\text { shotgun (5) }\end{array}$ \\
\hline & $\begin{array}{l}\text { Gastric cancer } \\
\text { and } \\
\text { adenocarcinoma }\end{array}$ & $\begin{array}{l}\mathrm{CN}, \mathrm{KR}, \\
\mathrm{MN}\end{array}$ & 6 & 498 & 467 & $50-58$ & $16 s$ \\
\hline & $\begin{array}{l}\text { Pancreatic } \\
\text { cancer and } \\
\text { ductal } \\
\text { adenocarcinoma }\end{array}$ & CN, US & 3 & 76 & 58 & $55-68$ & $16 s$ \\
\hline & Prostate cancer & US & 2 & 26 & 24 & $63-66$ & $\begin{array}{l}16 s(1) \text { and } \\
\text { shotgun (1) }\end{array}$ \\
\hline & Cervical cancer & CN, US & 2 & 50 & 51 & $48-60$ & $16 s$ \\
\hline & Lung cancer & $\mathrm{CN}$ & 1 & 30 & 30 & $50-61$ & $16 s$ \\
\hline & $\begin{array}{l}\text { Thyroid papillary } \\
\text { cancer }\end{array}$ & $\mathrm{CN}$ & 1 & 34 & 40 & $46-48$ & $16 s$ \\
\hline
\end{tabular}




\begin{tabular}{llllllll}
\hline & Breast cancer & CN & 1 & 43 & 90 & $49-51$ & shotgun \\
\hline $\begin{array}{l}\text { Hepatocellular } \\
\text { carcinoma (liver } \\
\text { cancer) }\end{array}$ & $\mathrm{CN}$ & 1 & 75 & 75 & $48-49$ & $16 \mathrm{~s}$ \\
\hline GIT neoplasia & $\mathrm{FI}$ & 1 & 63 & 13 & $43-70$ & $16 \mathrm{~s}$ \\
\hline Total & - & 27 & $84^{\star *}$ & 4208 & 5957 & - & - \\
\hline
\end{tabular}

*Arthritis studies include Ankylosing spondylitis $(n=1)$, Enthesitis-related arthritis $(n=2)$, Juvenile idiopathic arthritis $(n=1)$, Rheumatoid Arthritis $(n=5)$, and Spondyloarthritis $(n=1))$; **Two studies investigated two different diseases.

Country- AT (Austria), AU (Australia), AZ (Azerbaijan), BE (Belgium), CA (Canada), CN (China), DE (Germany), DK (Denmark), ES (Spain), FI (Finland), FR (France), IN (India), IR (Iran), IT (Italy), JO (Jordan), JP (Japan), KR (Republic of Korea), MA (Morocco), MN (Mongolia), MX (Mexico), NG (Nigeria), NL (Netherlands), PL (Poland),

SD (Sudan), SE (Sweden), UK (United Kingdom), US (United States of America).

\section{Figures}

\section{Figure 1}

PRISMA flow diagram of study selection.

\section{Figure 2}

Summary of the results of risk of bias assessment by the Newcastle-Ottawa Scale (NOS).

\section{Figure 3}

Alpha diversity and genus-level association of microbiome in cancer and autoimmune diseases. a) Trend of alpha diversity of microbiome in cancer and autoimmunity across studies. 'Decrease' and 'increase' indicate statistical significance $(p<0.05)$ difference of alpha diversity between cases and controls, and 'no difference' indicate no statistical significance $(p>0.05)$ difference of alpha diversity between cases and controls. b) Bacterial genera found to be significantly $(p<0.05)$ increased or decreased in cancer or 
autoimmune patients in multiple studies, c-h) Forest plot of log fold-change of relative abundance of six genera in cancer or autoimmune patients compared with healthy controls.

\section{Figure 4}

Species-level association and predicted metabolic pathways of microbiome in cancer and autoimmune diseases. Bacterial species (a) and predicted metabolic pathways (b) found to be significantly $(p<0.05)$ increased or decreased in cancer or autoimmune patients in multiple cancer and autoimmune studies.

\section{Figure 5}

Taxonomic and functional diversity of cancer and autoimmune associated microbiome. Taxonomic relatedness of bacterial genus (a) and species (b) in cancer and autoimmune diseases. The bars represent the total number of studies where the respective taxa were found to be significantly increased or decreased. *Fusobacteria phylum and **Verrucomicrobia phylum. The heatmap (c) shows 320 distinct metabolic pathways that were exclusively found either in cancer or autoimmune increased taxa. The pathways were predicted from a list of cancer and autoimmune microbiomes by querying the metaCyc database using MACADAM[105]. The color scale shows pathway completeness score. Pathway completeness score 1 indicates the respective bacterial taxa contains a full set of metabolic potentials to complete the pathway. The left cluster is likely autoimmune-specific microbial pathways and the right big cluster is likely cancer-specific microbial pathways. A complete list of these pathways can be found in the Supplementary file 1. The scatterplot (d) shows the number of pathways (dot size) with specific pathway score features between cancer and autoimmune microbes.

\section{Supplementary Files}

This is a list of supplementary files associated with this preprint. Click to download.

- SupplementaryTable1.docx

- SupplementaryTable2.doc

- SupplementaryTable3.docx

- SupplementaryTable4.docx

- SupplementaryTable5.docx

- SupplementaryFile10ld.xlsx 